\title{
A STRUCTURE THEOREM AND A SPLITTING THEOREM FOR SIMPLY-CONNECTED SMOOTH 4-MANIFOLDS
}

\author{
RichaRd STONG
}

\begin{abstract}
A вstract. We will prove that any closed, simply-connected smooth 4manifold admits a handlebody structure where the 2-handles homotopically cancel the 1-handles and the dual 1-handles in the nicest possible way. As a consequence we will derive the following improved splitting theorem for closed, simply-connected smooth 4-manifolds. Suppose $M$ is a closed, simply-connected, smooth 4-manifold and the intersection form of $M$ splits as $\left(H_{2}(M ; Z), q_{M}\right) \cong\left(Z^{n_{1}}, \lambda_{1}\right) \oplus\left(Z^{n_{2}}, \lambda_{2}\right)$. Then there is a decomposition $M \cong M_{1} \cup_{\Sigma} M_{2}$, where $M_{i}$ is a compact, simply-connected, smooth 4manifold with boundary the homology 3-sphere $\Sigma$ and intersection form isomorphic to $\left(Z^{n_{i}}, \lambda_{i}\right)$. This result is also extended to 4 -manifolds with free fundamental groups.
\end{abstract}

\section{Introduction}

In [3], M. Freedman and L. Taylor adapt Browder's high-dimensional splitting argument to dimension 4 for all fundamental groups. Specializing their result to the case of simply-connected 4-manifolds gives the following splitting theorem. Suppose $M$ is a closed, simply-connected, smooth 4manifold and the intersection form of $M$ splits as

$$
\left(H_{2}(M ; Z), q_{M}\right) \cong\left(Z^{n_{1}}, \lambda_{1}\right) \oplus\left(Z^{n_{2}}, \lambda_{2}\right)
$$

Then there is a decomposition $M \cong M_{1} \cup_{\Sigma} M_{2}$, where $M_{i}$ is a compact, smooth 4-manifold with boundary the homology 3 -sphere $\Sigma, H_{1}\left(M_{i} ; Z\right)=0$ and the intersection form is isomorphic to $\left(Z^{n_{i}}, \lambda_{i}\right)$. However by their argument it is impossible to conclude that the $M_{i}$ are 1-connected. We will show how to arrange this extra property; the result is the following theorem.

1991 Mathematics Subject Classification. 57N13.

Key words and phrases. 4-manifolds, handlebodies, splitting.

Received April 15, 1995.

Partially supported by an NSF postdoctoral fellowship and a Sloan fellowship. 
Theorem 1. Suppose $M$ is a closed, simply-connected, smooth 4-manifold and the intersection form of $M$ splits as

$$
\left(H_{2}(M ; Z), q_{M}\right) \cong\left(Z^{n_{1}}, \lambda_{1}\right) \oplus\left(Z^{n_{2}}, \lambda_{2}\right)
$$

Then there is a decomposition $M \cong M_{1} \cup_{\Sigma} M_{2}$, where $M_{i}$ is a compact, 1 connected, smooth 4-manifold with boundary the homology 3-sphere $\Sigma$ and intersection form isomorphic to $\left(Z^{n_{i}}, \lambda_{i}\right)$.

This theorem is an easy corollary of the following interesting structural claim for closed 1-connected smooth 4-manifolds. Suppose $\mathcal{H}$ is a handlebody structure on a smooth 4-manifold $M$. Let $M^{(i)}$ denote the union of the handles of dimension at most $i$, which we will refer to as the $i$-skeleton of $M$. Dually, let $\bar{M}^{(i)}$ denote the union of the handles of dimension at least $4-i$, thought of as the $i$-skeleton of the dual handlebody structure on $M$. We will call this the dual $i$-skeleton of $M$.

Theorem 2. Suppose $M$ is a closed 1-connected smooth 4-manifold. Then $M$ admits a handlebody structure $\mathcal{H}$ with 2-handles $\left\{H_{1}, H_{2}, \ldots, H_{m}\right\}$ such that

(1) The attaching circles for the handles $H_{1}, \ldots, H_{r}$ represent a free basis for $\pi_{1}\left(M^{(1)}\right)$ and the attaching circles for the other 2-handles are null-homotopic in $\pi_{1}\left(M^{(1)}\right)$.

(2) The boundaries of the cocores for $H_{r+1}, H_{r+2}, \ldots, H_{r+s}$ represent a free basis for $\pi_{1}\left(\bar{M}^{(1)}\right)$ and the boundaries of the cocores for the other 2-handles are null-homotopic in $\pi_{1}\left(\bar{M}^{(1)}\right)$.

Weaker (and more technical) forms of theorems 1 and 2 can be proven using the same techniques for 1-connected, smooth 4-manifolds with boundary. A result similar to theorem 2 appears as part of the author's contribution to [2].

It is worth noting that Freedman and Taylor also prove an analogous splitting theorem for 4-manifolds with arbitrary fundamental group [3]. Their statement goes as follows. Suppose we are given a smooth 4-manifold $(N, \partial N)$. Also suppose we have a Poincare duality pair, $(X, \partial X)$, which splits as $X=X_{1} \cup_{Y_{0}} X_{2}$ where $\left(X_{1}, \partial X_{1}\right)$ and $\left(X_{2}, \partial X_{2}\right)$ are also Poincare duality pairs, $Y_{0}$ is a component of $\partial X_{1}$ and $\partial X_{2}$ and $\pi_{1}\left(Y_{0}\right) \rightarrow \pi_{1}\left(X_{i}\right)$ is an isomorphism for $i=1$ or 2 . Finally suppose we have a $Z\left[\pi_{1}(X)\right]$-homology equivalence $(N, \partial N) \rightarrow(X, \partial X)$. With these hypotheses Freedman and Taylor construct a splitting of $N$ up to $Z\left[\pi_{1}(X)\right]$-homology equivalence. We are interested in generalizing the results of [3] only in the special case where $N$ is closed and $\pi_{1}(N)=\pi_{1}(X)$. With these extra hypotheses, the 
fundamental group $\pi=\pi_{1}(X)=\pi_{1}(N)$ is severely restricted. For example it follows from [1, Lemma 3.2] that if $Y_{0}$ is actually a 3-manifold, then $\pi$ must be free. It would seem reasonable to conjecture that this conclusion holds even if $Y_{0}$ is only a Poincare duality space. With this in mind, the obvious extension of theorems 1 and 2 to smooth 4-manifolds with free fundamental groups is sketched after the proofs of theorems 1 and 2 .

Proofs. We now turn to the task of proving theorem 2. Suppose $M$ is a closed, oriented, 1-connected, smooth 4-manifold. Fix a handlebody structure $\mathcal{H}$ on $M$ with only one 0 -handle and only one 4 -handle. We would like to fix a little extra notation for $\mathcal{H}$. Let $Z=M^{(1)} \cap \bar{M}^{(1)}$. Note that we can also describe $Z$ as $\partial M^{(1)}$ with the interiors of the attaching regions for the 2 -handles removed, or dually as $\partial \bar{M}^{(1)}$ with open regular neighborhoods of the boundary of the cocores of the 2-handles removed. In particular, the inclusion maps $\pi_{1}(Z) \rightarrow \pi_{1}\left(\partial M^{(1)}\right) \rightarrow \pi_{1}\left(M^{(1)}\right)$ and $\pi_{1}(Z) \rightarrow \pi_{1}\left(\partial \bar{M}^{(1)}\right) \rightarrow \pi_{1}\left(\bar{M}^{(1)}\right)$ are onto. Denote these two compositions by $p$ and $\bar{p}$. If $\alpha \in \pi_{1}(Z)$ and $x \in \pi_{1}\left(M^{(1)}\right)$ or $\pi_{1}\left(\bar{M}^{(1)}\right)$, let $x^{\alpha}=\alpha x \alpha^{-1}$ denote the result of letting $\alpha$ act on $x$ by conjugation. Pick a base point in $Z$ and choose arcs joining each 2-handle of $\mathcal{H}$ to this base point. Then for each 2-handle $H_{i}$ of $\mathcal{H}$, we can associate a pair of group elements $\left(g_{i}, h_{i}\right)$, where $g_{i}$ is the image of the attaching curve of $H_{i}$ in the free group $\pi_{1}\left(M^{(1)}\right)$ and $h_{i}$ is the image of the boundary of the cocore of $H_{i}$ in the free group $\pi_{1}\left(\bar{M}^{(1)}\right)$. Thus to any handlebody structure we assign a sequence of pairs of group elements $\left\{\left(g_{i}, h_{i}\right)\right\}$. With this extra terminology, theorem 2 may be rephrased as follows:

Claim. By handle passes, stabilization and reordering handles, we may arrange that

(1) $g_{1}, \ldots, g_{r}$ are free generators for $\pi_{1}\left(M^{(1)}\right)$,

(2) $g_{r+1}, \ldots, g_{n}$ are trivial,

(3) $h_{r+1}, \ldots, h_{r+s}$ are free generators for $\pi_{1}\left(\bar{M}^{(1)}\right)$, and

(4) $h_{1}, \ldots, h_{r}, h_{r+s+1}, \ldots, h_{n}$ are trivial.

Of course after a stabilization one of the groups $\pi_{1}\left(M^{(1)}\right)$ or $\pi_{1}\left(\bar{M}^{(1)}\right)$ will have higher rank. However, we continue to use the same notation for the enlarged group. This should not cause undue confusion.

To establish this claim we first must understand the effect of a handle pass of handle $H_{i}$ over $H_{j}$. To perform such a handle pass, we must first choose a path $\gamma$ in $Z$ joining $H_{i}$ to $H_{j}$. Suppose the path from the base point to $H_{i}$, over $\gamma$ to $H_{j}$ and back to the base point represents the element $\alpha \in \pi_{1}(Z)$. Then, in terms of the $g$ 's, the effect of passing $H_{i}$ over $H_{j}$ along 
$\gamma$ is to replace $g_{i}$ by $g_{i}{ }^{\prime}=g_{i}\left(g_{j}\right)^{\alpha}$. In the dual picture, we see the handle dual to $H_{j}$ being passed over the handle dual to $H_{i}$ with the reversed orientation along the reverse path to $\gamma$. Therefore the effect on the $h$ 's is to replace $h_{j}$ by $h_{j}{ }^{\prime}=h_{j}\left(h_{i}{ }^{-1}\right)^{\alpha^{-1}}$.

The action of $\alpha$ on $\pi_{1}\left(M^{(1)}\right)$ by conjugation is the same as the action of $p(\alpha)$ on $\pi_{1}\left(\bar{M}^{(1)}\right)$ by conjugation, and analogously for $\pi_{1}\left(M^{(1)}\right)$. Hence in each case we are adding a conjugate of one handle to the other. We would like to know that we can choose these conjugates independently. That is the content of the following lemma.

Lemma. The homomorphism $p \times \bar{p}: \pi_{1}(Z) \rightarrow \pi_{1}\left(M^{(1)}\right) \times \pi_{1}\left(\bar{M}^{(1)}\right)$ is onto.

Proof. Look at a meridian $m_{i}$ to the $i$ th 2-handle as a curve in $Z$. Unwinding the definitions, $p\left(m_{i}\right)=1$ and $\bar{p}\left(m_{i}\right)=h_{i}$. Thus, $\left(1, h_{i}\right)$ is in the image of $p \times \bar{p}$. The projection $\pi_{1}(Z) \rightarrow \pi_{1}\left(\bar{M}^{(1)}\right)$ is onto. Hence any conjugate $\left(1, h_{i}{ }^{y}\right)$ of $\left(1, h_{i}\right)$ is also in the image of $p \times \bar{p}$. Since the $h_{i}$ are a normal generating set for $\pi_{1}\left(\bar{M}^{(1)}\right),\{1\} \times \pi_{1}\left(\bar{M}^{(1)}\right)$ is in the image of $p \times \bar{p}$. Similarly, $\pi_{1}\left(M^{(1)}\right) \times\{1\}$ is in the image and $p \times \bar{p}$ is onto.

Corollary. For any $i \neq j, x \in \pi_{1}\left(M^{(1)}\right)$, and $y \in \pi_{1}\left(\bar{M}^{(1)}\right)$, we may perform a handle pass to replace $g_{i}$ by $g_{i}{ }^{\prime}=g_{i}\left(g_{j}\right)^{x}$ and $h_{j}$ by $h_{j}{ }^{\prime}=h_{j}\left(h_{i}{ }^{-1}\right)^{y}$ while leaving all the other group elements the same.

For general fundamental groups, the proof of the lemma adapts to give that

$$
p \times \bar{p}: \pi_{1}(Z) \rightarrow\left\{(g, h) \in \pi_{1}\left(M^{(1)}\right) \times \pi_{1}\left(\bar{M}^{(1)}\right) \mid g \text { and } h \text { agree in } \pi_{1}(M)\right\}
$$

is onto. Then we get an analogous form of the corollary with the added hypothesis that $x$ and $y$ represent inverse elements of $\pi_{1}(M)$.

Now consider the effect of doing two such moves in a particularly nice pattern. First we pass $H_{i}$ over $H_{j}$ along a path which conjugates by $x \in$ $\pi_{1}\left(M^{(1)}\right)$ and by $y=1 \in \pi_{1}\left(\bar{M}^{(1)}\right)$. The result is to make $g_{i}{ }^{\prime}=g_{i}\left(g_{j}\right)^{x}$ and $h_{j}{ }^{\prime}=h_{j}\left(h_{i}{ }^{-1}\right)$ and no other changes. Next we pass $H_{i}$ over $H_{j}$ with the reversed orientation along a path which conjugates by $x a \in \pi_{1}\left(M^{(1)}\right)$ and by $\mathrm{y}=1 \in \pi_{1}\left(\bar{M}^{(1)}\right)$. The result is to make $g_{i}{ }^{\prime \prime}=g_{i}\left(g_{j}\right)^{x}\left(g_{j}{ }^{-1}\right)^{x a}=$ $g_{i}\left[g_{j}, a\right]^{x}$ and $h_{j}{ }^{\prime \prime}=h_{j}\left(h_{i}{ }^{-1}\right) h_{i}=h_{j}$. Thus we have changed only one group element by a conjugate of a commutator and have left all other group elements the same. Note that for general fundamental groups this two-move construction still works provided that $a$ is trivial in $\pi_{1}(M)$. The only change is that one must allow $y$ to be nontrivial but equal in the two moves. 
It is now an easy exercise to use the operations to produce the desired set of 2-handles. By adding trivial 1-, 2-handle pairs and performing handle passes (basically ignoring the $g$ 's), one can arrange that $h_{1}, \ldots, h_{n}$ are free generators for $\pi_{1}\left(\bar{M}^{(1)}\right)$ and that $h_{n+1}=h_{n+2}=\cdots=1$. The group elements $g_{n+1}, g_{n+2}, \ldots$ must still form a generating set for the abelianization $\pi_{1}\left(M^{(1)}\right) /\left[\pi_{1}\left(M^{(1)}\right), \pi_{1}\left(M^{(1)}\right)\right]=H_{1}\left(M^{(1)} ; Z\right)$. (In fact, since $\partial \circ \partial=0$ in the cellular chain complex of $M$, we see that $g_{1}, \ldots, g_{n}$ must vanish in $H_{1}\left(M^{(1)} ; Z\right)$.) Therefore, by handle passes, among only the last block of handles, we may assume $g_{n+1}, \ldots, g_{n+r}$ are the standard generators of $H_{1}\left(M^{(1)} ; Z\right)$ and $g_{n+r+1}, g_{n+r+2}, \ldots$ are null-homologous. Thus there are products of commutators $c_{1}, \ldots, c_{r}$ such that $\left\{g_{n+i} c_{i}\right\}$ is the standard free basis for $\pi_{1}\left(M^{(1)}\right)$.

Stabilize by adding $r$ more 2-, 3-handle pairs. These 2-handles have group elements $\left(1, e_{i}\right)$, where the $e_{i}$ are the new free generators of the enlarged $\pi_{1}\left(\bar{M}^{(1)}\right)$. By the two step move described above, we may add any conjugate of any commutator with any $g_{i}$ to the $g$ term of any of these 2 -handles. The $g_{i}$ are a normal generating set for $\pi_{1}\left(M^{(1)}\right)$. Hence by a sequence of such moves, we may build up any element of $\left[\pi_{1}\left(M^{(1)}\right), \pi_{1}\left(M^{(1)}\right)\right]$. Therefore, we may replace the 2 -handles representing $\left(1, e_{i}\right)$ by 2 -handles representing $\left(c_{i}, e_{i}\right)$. Adding a copy of the handle representing $\left(c_{i}, e_{i}\right)$ to the handle representing $\left(g_{n+i}, 1\right)$ will give us handles representing $\left(g_{n+i} c_{i}, 1\right)$ and $\left(c_{i}, e_{i}\right)$. At this point we have the following. We have one set of 2handles representing $\left(x_{i}, 1\right)=\left(g_{n+i} c_{i}, 1\right)$ where the elements $x_{i}=g_{n+i} c_{i}$ are a free generating set for $\pi_{1}\left(M^{(1)}\right)$. A second set of 2-handles represent $\left(g_{i}, y_{i}\right)=\left(g_{i}, h_{i}\right)$ and $\left(c_{i}, y_{i+n}\right)=\left(c_{i}, e_{i}\right)$, where the elements $y_{i}$ are a free generating set for $\pi_{1}\left(\bar{M}^{(1)}\right)$. Finally we have some additional leftover 2 -handles both of whose group elements are $\left(g_{i}, 1\right)$. Note that the extra $g_{i}$ and the $c_{i}$ are all products of commutators in $\pi_{1}\left(M^{(1)}\right)$. Using the two step move described above we may use the handles representing $\left(x_{i}, 1\right)$ to kill off the extra $g_{i}$ and the $c_{i}$, obtaining handles representing $\left(1, y_{i}\right)$ and $(1,1)$.

This completes the proof of the structure theorem for handle decompositions. Theorem 1 now follows immediately. Suppose $M$ is a closed, simply-connected, smooth 4-manifold and the intersection form of $M$ splits as $\left(H_{2}(M ; Z), q_{M}\right) \cong\left(Z^{n_{1}}, \lambda_{1}\right) \oplus\left(Z^{n_{2}}, \lambda_{2}\right)$. Choose a handlebody structure on $M$ as guaranteed by theorem 2 . We may identify $H_{2}(M ; Z)$ with the free abelian group on the 2 -handles representing trivial group elements. By handle passes among these we may arrange that the first $n_{1}$ of these 2-handles represent a basis for the first summand and the remaining $n_{2}$ represent a basis for the second summand. Let $M_{1}$ be the union of the 
$M^{(1)}$, the 2-handles representing a free basis for $\pi_{1}\left(M^{(1)}\right)$ and the first $n_{1}$ 2-handles representing trivial group elements. Note that $\partial M^{(1)}$ must be some homology 3 -sphere $\Sigma$. Let $M_{2}$ be the union of the remaining handles, namely $\bar{M}^{(1)}$, the 2-handles representing a free basis for $\pi_{1}\left(\bar{M}^{(1)}\right)$ and the last $n_{2}$ 2-handles representing trivial group elements. Then we have a decomposition $M \cong M_{1} \cup_{\Sigma} M_{2}$, where $M_{i}$ is a compact, 1-connected, smooth 4-manifold with boundary the homology 3 -sphere $\Sigma$ and intersection form isomorphic to $\left(Z^{n_{i}}, \lambda_{i}\right)$.

The extension of these results to manifolds with free fundamental group is straightforward and goes as follows. Suppose $M$ is a closed, smooth 4manifold with free fundamental group $F_{k}$. Fix a handlebody structure $\mathcal{H}$ on $M$. We may easily arrange that $\mathcal{H}$ has only one 0 -handles and only one 4-handles. Furthermore we can arrange that the first $k$ 1-handles represent the standard free basis for $F_{k}$ and that the remaining $m$ 1-handles represent the identity and similarly for the 3-handles (dual 1-handles). Then the kernel of the map $\pi_{1}\left(M^{(1)}\right) \rightarrow \pi_{1}(M)=F_{k}$ is normally generated by loops homotopic to the cores of the last $m$ 1-handles and these $m$ elements represent a free basis for $H_{1}\left(M^{(1)} ; Z\left[F_{k}\right]\right)$ as a $Z\left[F_{k}\right]$-module. Now the proof proceeds exactly as above, replacing $Z$ coefficients with $Z\left[F_{k}\right]$ where necessary. The result is the following structure theorem.

Theorem 3. Suppose $M$ is a closed, smooth 4-manifold with free fundamental group $F_{k}$. Then $M$ admits a handlebody structure $\mathcal{H}$ with 2-handles $1, H_{2}, \ldots, H_{m}$ such that

(1) The first $k$ 1-handles represent the standard free generating set for $\pi_{1}(M)$. The remaining $r$ 1-handles represent the identity in $\pi_{1}(M)$ and the attaching circles for $H_{1}, \ldots, H_{r}$ are homotopic to the cores of these $r$ 1-handles in $\pi_{1}\left(M^{(1)}\right)$. The attaching circles for the other 2-handles are null-homotopic in $\pi_{1}\left(M^{(1)}\right)$.

(2) The first $k$ 3-handles represent the standard free generating set for $\pi_{1}(M)$. The remaining $s$ 3-handles represent the identity in $\pi_{1}(M)$; boundaries of the cocores for $H_{r+1}, H_{r+2}, \ldots, H_{r+s}$ are homotopic to the cocores of these $r$-handles in $\pi_{1}\left(\bar{M}^{(1)}\right)$. The boundaries of the cocores for the other 2-handles are null-homotopic in $\pi_{1}\left(\bar{M}^{(1)}\right)$.

As a consequence we get the following splitting theorem for 4-manifolds with free fundamental group, which improves on the splitting theorem of Freedman and Taylor [3] in this case. 
Theorem 4. Suppose $M$ is a closed, oriented, smooth 4-manifold with free fundamental group $F_{k}$ and the intersection form of $M$ splits as

$$
\left(\pi_{2}(M), q_{M}\right) \cong\left(Z\left[F_{k}\right] n_{1}, \lambda_{1}\right) \oplus\left(Z\left[F_{k}\right] n_{2}, \lambda_{2}\right)
$$

Then there is a decomposition $M \cong M_{1} \cup_{\Sigma} M_{2}$, where $M_{i}$ is a compact, smooth 4-manifold with fundamental group $F_{k}$, intersection form isomorphic to $\left(Z\left[F_{k}\right] n_{i}, \lambda_{i}\right)$ and boundary $\Sigma$, where $\Sigma$ has the $Z\left[F_{k}\right]$-homology of $\#^{k}\left(S^{1} \times S^{2}\right)$.

\section{Acknowledgements}

The author would like to thank Mike Freedman and Tim Cochran for their assistance.

\section{References}

1. T. Cochran, Ribbon Knots in S4, J. London Math. Soc.(2) 28 (1983), 563-576.

2. C. Curtis, M. Freedman, W. C. Hsiang and R. Stong, A decompositon theorem for h-cobordant smooth simply-connected compact 4-manifolds, Invent. Math. (to appear).

3. M. Freedman and L. Taylor, L-Splitting 4-manifolds, Topology 16 (1977), 181-184.

Departament of Mathematics, Rice University, Houston, TX 77251

E-mail address: stong@math.rice.edu 